\title{
An Android Mobile-based Measurement Application - Object and Interior Room Measurement App
}

\author{
https://doi.org/10.3991/ijim.v14i20.15415 \\ Elcy Tsen Yi Ching, Chin Kim On $\left.{ }^{\bowtie}\right)$, Rayner Alfred, \\ Mohd Hanafi Ahmad Hijazi \\ Universiti Malaysia Sabah, Kota Kinabalu, Malaysia \\ kimonchin@ums. edu.my \\ Tan Tse Guan \\ Universiti Kelantan Malaysia, Bachok, Malaysia
}

\begin{abstract}
The conventional and most common way to measure the interior of a room is by using a measuring tape and other similar tools. However, it is not productive, and often more than one worker is required to assist the measurement process. The goal of this study is to discuss the acceptability and usability of an Android-based measurement mobile app designed for interior designers, civil engineer designers, and water pipe workers. The user can measure the traditional form of the interior room from the image captured and to give the result of the area of space from the measurement taken from the image. The developed mobile app includes a virtual screen ruler to measure the approximate length and width of the room. It can be achieved by having a reference scale from the user, and the camera lens must be focused on the study points. The result is then calculated from the video stream through the camera. The data obtained is then formulated in the area in order to obtain the result of the space area. The user will receive the results on the display screen as soon as the calculation is carried out. Later, the results revealed that, while not part of the original research goal, the applications can also be used to calculate the height and length of an object. Finally, an extensive review of the mobile app is provided, which shows its high applicability and user acceptability.
\end{abstract}

Keywords-Information Systems, Information Science, Mobile App Design, Design Science Approach, Measurement App

\section{Introduction}

The advancement in the ability of today's smartphones has taken off tremendously. It has also allowed us to introduce a new methodology that enables mobile device sensors/tools to be used to capture, measure, and track almost everything around a person or an entity. Most mobile devices have cameras (front and back), GPS antenna, NFC antenna, fingerprint detection sensor, iris recognition sensor, proximity sensors (detector and emitter), etc. [2][7]. Thousands of applications have been developed due to the availability of free sensors. Mobile programmers have created measuring apps 
such as pedometers that count each step a person takes by monitoring the motion of a person [44], speedometers or odometer apps to measure car and bike speed [41], pulse apps to measure user pulse rate by analyzing blood flow information from fingertip [25], tape measurement apps to measure length, distance, location, height, or even steps taken by the user, etc. [20][36]. Apps are a common demand in the technology industry in this era of globalization, and maybe one day, there will be smartphone app available to detect viruses/bacteria in our blood.

As far as we are concerned, there are cases in which a person fails to remember to bring his measuring tape with him or has never planned to bring any measuring tools at that time. Professionals such as site contractors, interior designers, architects, engineers, and more may need to measure a room at random. The biggest challenge that these professional professions can face is that they do not have the tape to measure with them all the time. The tool that one will bring with them all the time, however, would be their smartphone. That being the case, the mobile app will make measurements possible. There are certainly hundreds of current mobile measurement apps that can offer real-time length and width or offer measurement from an image. However, there are not many smartphone apps that are user-friendly yet, and most of the available apps required high-end smartphones as augmented reality technology is used [27]. In reality, most site contractors, interior designers, engineers, and pipe workers are not affordable for high-end smartphones.

On the other hand, many measuring systems cause inconvenience compared to the conventional measuring tape, where one can easily pull the tap across the room and receive measurement without further adjustment. Nonetheless, in some situations, it is not efficient, and more than one person is often needed to assist the measurement process. Therefore, it motivates us to create an easy to use, low-end, supportable measurement app to help local site contractors, interior designers, and pipe workers with their daily tasks.

This paper presents the creation of an Android-based measurement app. It is a virtual measuring tool to obtain the estimated length and width of the room by obtaining a reference scale from the user. The camera lens must be directed at the points under review. The result is then calculated and immediately showed on the screen. In addition, the object measurement function has also been introduced. The proposed app can calculate the length or height of an item by entering the defined reference scale or custom scales, such as a coin or credit card.

Furthermore, the analysis of requirements, the framework, and the critical implementation problems will be addressed by relevant UML charts. The functionality of the program is also displayed through screenshots and concise descriptions. We developed a questionnaire from which we were able to test the application and see if the proposed app meets the specific user needs in terms of usability and functionality. The findings of this assessment are also discussed. Finally, future changes are discussed, and closing remarks are made. 


\section{Related Works}

The essential features of selected Android measuring apps available in the Google Play Store are presented in this section. The selection of these apps was based on the features they have, their visual design, and because they are some of the popular apps (scored 4-star user review) found in the Google Play store. Apps developed on the iOS platform are not being thoroughly reviewed as our project focuses on Androidbased app development.

In this section, the conventional ruler app is first discussed in Section 2.1. Then, the discussion is followed by 2D photo measurement apps in Section 2.2. Sections 2.3 and 2.4 describe the real-time ruler apps and Global Positioning Services (GPS) rule apps, respectively. Next, the discussion is followed with the augmented reality-based rule app in Section 2.5, and lastly, the angle-based measurement app is described in Section 2.6

\subsection{Conventional ruler apps}

[31][32][37][45] are examples of traditional ruler apps. These ruler apps are apps that turn Android's smartphone into a nifty ruler. These apps simply show a virtual ruler on the screen with correct markings. In using it, the user needs to place the phone straight down and then place the object at the top of the phone and measure it as if it was using the actual ruler. Users can choose the "caliper mode" in which they can shift the two handles that appear on the screen to measure them more precisely. Furthermore, the calibration can be modified or converted to suit various measurement needs by setting the units to a centimeter, millimeter, inch, or more. However, this feature depending on whether the apps are free or paid version. It is also available in a few different measurement modes: point, line, plane, and degree. However, only certain full version apps can save the measurement data. The biggest drawback of these apps is the length of the smartphone used.

\subsection{D Photo measurement apps}

The [8][12][17] allows the user to take measurements on an existing image instead of using a phone camera. The apps are not real-time measurement tools because it uses an image to do all its measurement. It is impossible for a regular camera to capture and return the measurement result from an image without using reference points to make further measurements. The app automatically compensates the measurements and angles for potential distortions in perspective. The complete version of [12] includes additional functions to calculate the angle and area of the image. The user can also insert text and drawing in the apps. 


\subsection{Real-time ruler apps}

[12][16][21][38] are measuring apps that measure an object using the smartphone camera. The camera lets the app measure the distance of the object and the height of the object. The estimation of the measurement is based on the height of the camera and the tilt of the screen. Thus, the user needs to ensure that he/she first set the appropriate camera height and then moves the measurement box on the screen to match the object he/she is going to measure. The measurement value appears on the screen after the calculation is completed automatically. For [38], the user may change the height directly on the main screen, which is more convenient. But this app is less accurate.

\subsection{Ruler apps based on GPS}

By using GPS data, [13][24][26][30] are mainly used to measure large areas, especially helpful for farmers who have to design their farms, outdoor activity planners who work on-site planning, construction workers, and architects. It effectively uses existing GPS technology. These apps also can measure a distance using GPS data. The measurement result will be displayed by selecting the starting and ending points, and then click on the start measure button. In order to measure the area, the user must tap at least three points on the map. The only downside of this app is its reliability and accuracy. Furthermore, travel distance (route) measurement from a single point to another is not allowed.

\subsection{Ruler with augmented reality technology apps}

Using augmented reality technology, programmers have developed powerful measurement apps [18][19][35][43]. The apps allow the user to measure any object with a phone camera and tap a starting point to start measuring. The measurement will be displayed on each line as the user taps each point of the object. The measurement result is highly accurate. There are also several other important features that assist with measuring work in layout design or house decoration. The only drawback to this software is its hardware specifications. Not all types of Android smartphones support augmented reality technology.

\subsection{Angle measurement apps}

[1][14][28][34][39][40] are angle measurement apps that consist of more than one feature. Usually, they include an on-screen scale, a 2D rule for measuring both sides of the phone at once, a bubble level, and an angle meter utility that uses the camera to calculate object angles in the real world. The advantage of these apps is the all-in-one functionality, but the installer file size is five times higher than other measurement apps. Hence, loading time is another concern when the app is installed on a low-end smartphone. 


\subsection{Summary of review}

Most apps available in Google App Store have restricted functionality and are not free of charge. The study found that measuring apps with augmented reality technology provided the most reliable and precise measurement. Unfortunately, these apps have hardware compatibility issues and require high-end smartphones. Furthermore, most of the current measurement apps do not have a tutorial or guide for using the apps. Furthermore, only certain full versions of the measurement apps allow users to save data.

\section{$3 \quad$ Methodology}

The research methods adopted in the development process includes the following steps:

\subsection{Data collection and questionnaires}

Data from this study was obtained by distributing questionnaires offline to a sample of the pre-determined population. The target respondents were 12 interior designers, 23 site contractors, and 15 pipe workers. The survey was conducted with the consent of the respondents. The survey was split into pre-and post-process stages. Presurvey gathers user basic demography details and user requirements while post-survey captures users' input on the usability and functionality of the developed measurement app.

\subsection{Room measurement algorithm}

A triangular similarity algorithm can be used to find the distance between the camera and the object. The width of the image used is firstly defined, and then the reference point is marked on the object. The formula can be represented as:

$$
f=(P \times D) W
$$

Where, $f=$ focal length, $P=$ width in pixels, $D=$ distance of image to camera, and $W=$ width of image/object.

The focal value can be obtained by taking an image with the camera of the smartphone. When the camera moves closer or farther away from the target, the triangle similarity algorithm can be used to determine the distance of the target from the camera. The formula is shown below.

$$
D^{\prime}=(W \times f) P
$$

Where, $D^{\prime}=$ distance between camera and object, $f=$ focal length calculated in formula (1), $P=$ width in pixels, and $W=$ width of image/object.

The method to be used in this measurement app is by using a trigonometry algorithm to measure the distance between the user camera and the target point. The dis- 
tance between the user camera and the object can be calculated using the following distance formula.

$$
d=h \times \tan a
$$

Where $d$ is the distance, $h$ is the device height held by the user, and $\tan a$ is the angle of the device required to capture distance.

\subsection{Object measurement algorithm}

The image processing region growing approach is used in the object measuring function. A small area is iteratively combined according to similarity constraints. The region is developed from seed pixels by adding identical adjacent pixels, resulting in an increase in the size of the region. If the growth of one region stops, another region of seed pixel will start rising again. The region-growing approach is only applicable to one of the reference measurements, while custom measurements use the pixel ratio equation by comparing the real reference length and the coordination reference length, as shown in the formula.

$$
p r=r l / c d
$$

Where $p r$ is the pixel ratio, $r l$ is the reference length used by the user, and $c d$ is the coordinate reference length distance based on unit. The object is then calculated on the basis of the length of the reference taken, and the results are shown on the smartphone screen. The picture and its measurement can be stored in the internal gallery storage unit.

\subsection{Use case diagram}

This section addresses the method of interpreting data and the decomposition of its components depicted in the use case diagram, as shown in Figure 1. There is only a user entity involves in the app. Once the app begins, the user can select a tutorial / user manual, object measurement function, or interior room measurement function. When the user chooses a tutorial/user manual function, a list of user manuals will be shown. Next, users may either trigger the camera or load the image from the directory in the measuring object function. Later, the user can choose whether to save the measurement result. Users can retrieve saved data from the image load function. The camera function will be triggered automatically if the user wants to measure the interior of the room. The user may then either change the height and calculate the room or set the measurement unit first. The default unit of measurement is set to the meter. The user must set the height of the camera before the results are obtained for the distance, length, and area of the space. Then, the measurement result can be saved to the local smartphone directory by pressing the Save button. Measurement reading could be reset at any time, if necessary. All measurement readings can be seen in the History section. The unit used in the calculation result could be converted by the user. 


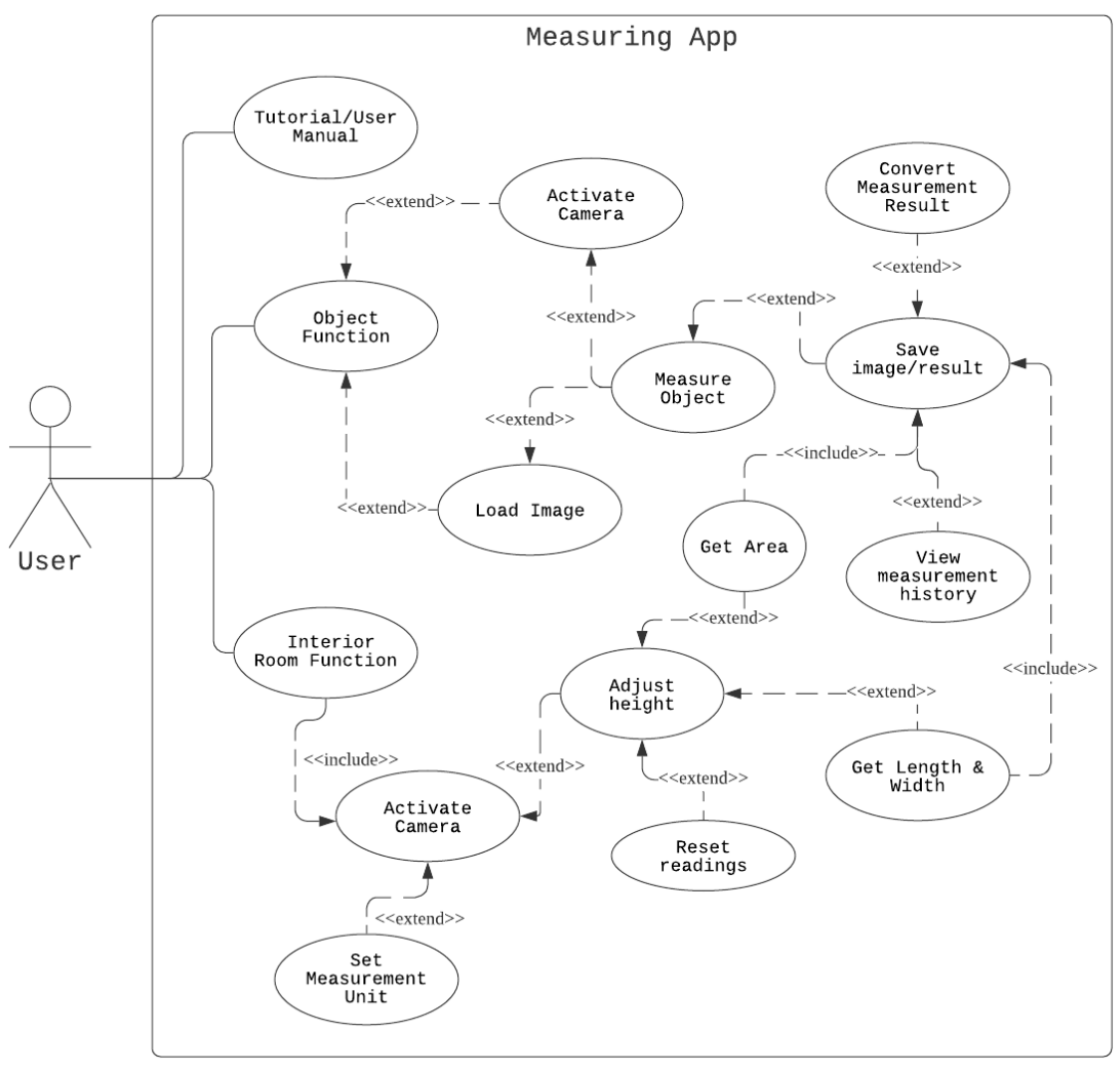

Fig. 1. The proposed measurement apps use case diagram

\subsection{Development technologies}

As discussed in Section 4.1 Requirement Analysis, an authentication feature is not required. Thus, a cloud-based database is not involved. Instead, a local text file is sufficient to save the reading of the measurement.

The Android Studio 3.6 Software Development Kit (SDK) [15] is used for development purposes. It functions as a cross-platform software development that reduces the complexity of finding the correct app or package. Later, the smartphone user should have at least version 5.0 of the Android OS and be fitted with 1 GB of RAM to use the measuring app smoothly.

\section{The Prototype Design}

This section provides an overview of the user requirements and a prototype demonstration of the developed measurement app. Pre-survey was performed to obtain an overview of the user requirements. Then, a post-survey was carried out to 
assess if it meets the user's expectations in terms of usability and functionality and the post-survey results will be discussed in Section 5.0.

\subsection{Requirement analysis}

The pre-survey analyses show that all female respondents are interior designers. Twenty-three male respondents were working as site contractors, fifteen male respondents are piping workers, and seven male respondents are interior designers. Clearly, there is much less female participation in these three types of occupations.

The analysis results also show that female respondents are between 21 and 40 years old. The majority of male respondents are between 41 and 50 years old. $20 \%$ of respondents are between 21 and 30 years old; they are very young and may have fewer working experiences. $16 \%$ of male respondents are between 31 and 40 years old, and only $6 \%$ are between 51 and 60 years old.

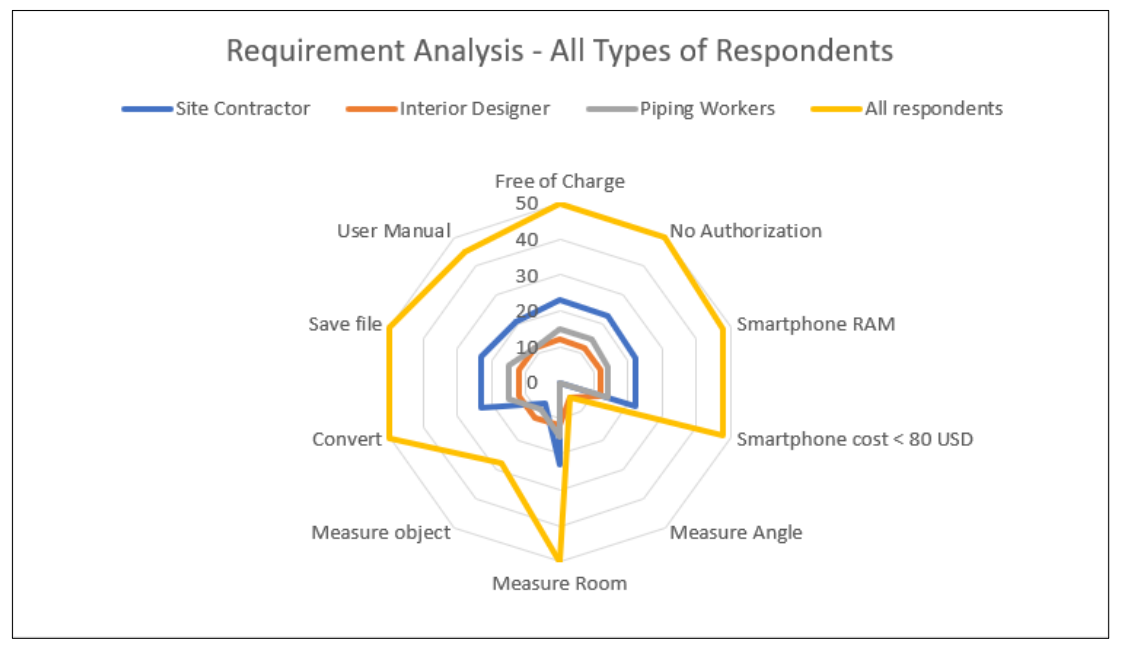

Fig. 2. Requirement Analysis for All Types of Respondents

Figure 2 has revealed that the measurement app that is being developed in this project should have the following features:

- A basic guideline or tutorial for using the app.

- Virtual measurement dependent.

- Measurement feature for interior design.

- Function to calculate the height or length of any object.

- Function to save the result of a measurement.

- Function for switching between the measurement units used. Units include centimeter, inch, foot, and meter.

- The function to convert readings to other measurement units. 
- Able to support low-end smartphones with real-time visual measurements. It requires a camera function with video support to exploit the video stream from the smartphone camera.

\subsection{Prototype demonstration}

Within this section, the most important interfaces and functions of the proposed measurement app's screenshots are shown and discussed. The interfaces are shown in Figures 3, 4, and 5, respectively.

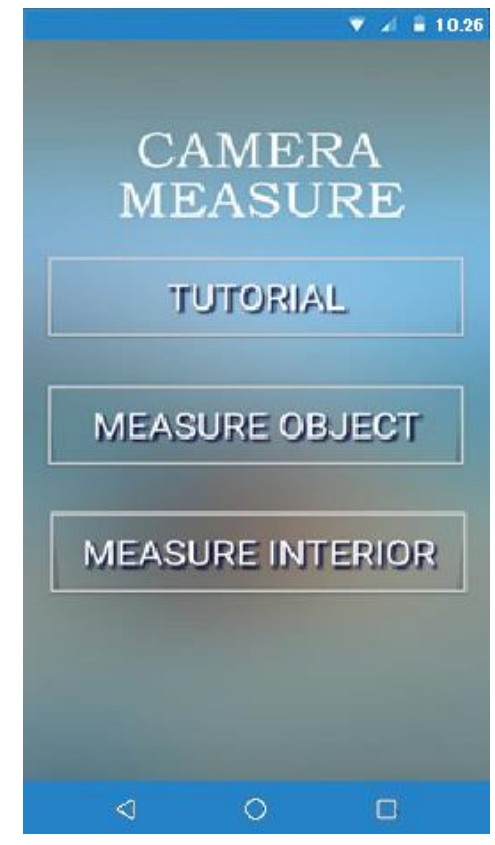

(a)

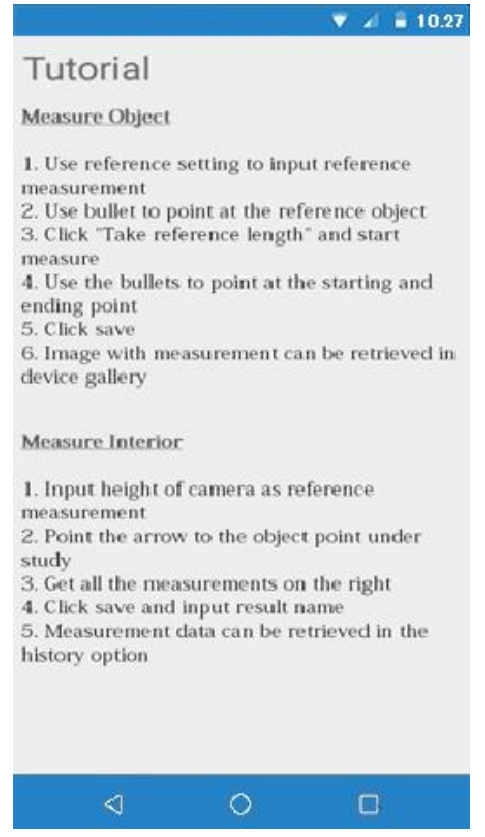

(b)

Fig. 3. Main Program Interface (a) and Tutorial Page (b)

The main measuring app page, as shown in Figure 3(a) will pop up once the user clicks the measurement app icon on their smartphone if the user has already installed the app. The main page has three buttons; a tutorial button, a measure object button, and a measure interior (room) button. The page will be flipped to the tutorial tab (Figure 3(b)) if the user clicks the tutorial button. A basic tutorial or guideline has been included in the tutorial tab. At first, we attempt to include a gamification feature to direct the user to the tutorial tab. Nevertheless, the gamification feature has significantly increased the size of the app, so we chose to keep a basic one-page tutorial tab. 


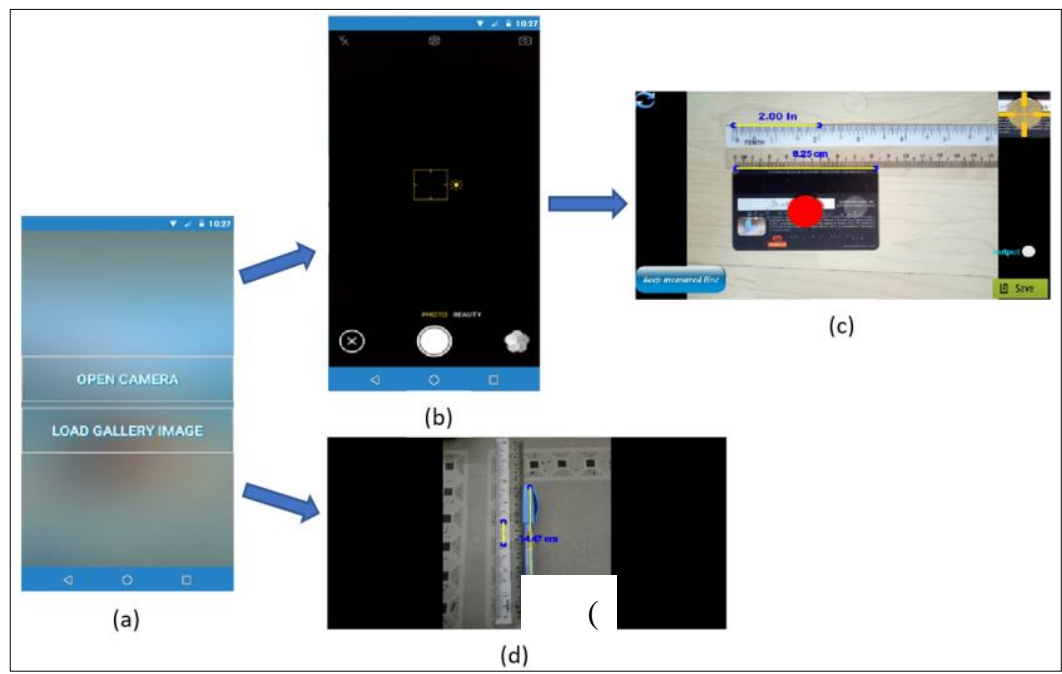

Fig. 4. Object Measurement Interfaces

The page would be flipped to Figure 4(a) if the user clicked the Measure Object button on the main page (Figure 3(a)). There are two buttons on the screen, where the user can either click the open camera button which will be guided to the screen shown in Figure 4(b) to measure the new object or click the Load gallery image button to measure any object in the picture as shown in Figure 4(d). Figure 6(c) indicates that a coin was used as a reference length for measuring a credit card. Figure 4(d) indicates that the custom reference point ( 1 inch of rule) was used to measure the length of the pen, and the app has returned $14.47 \mathrm{~cm}$.

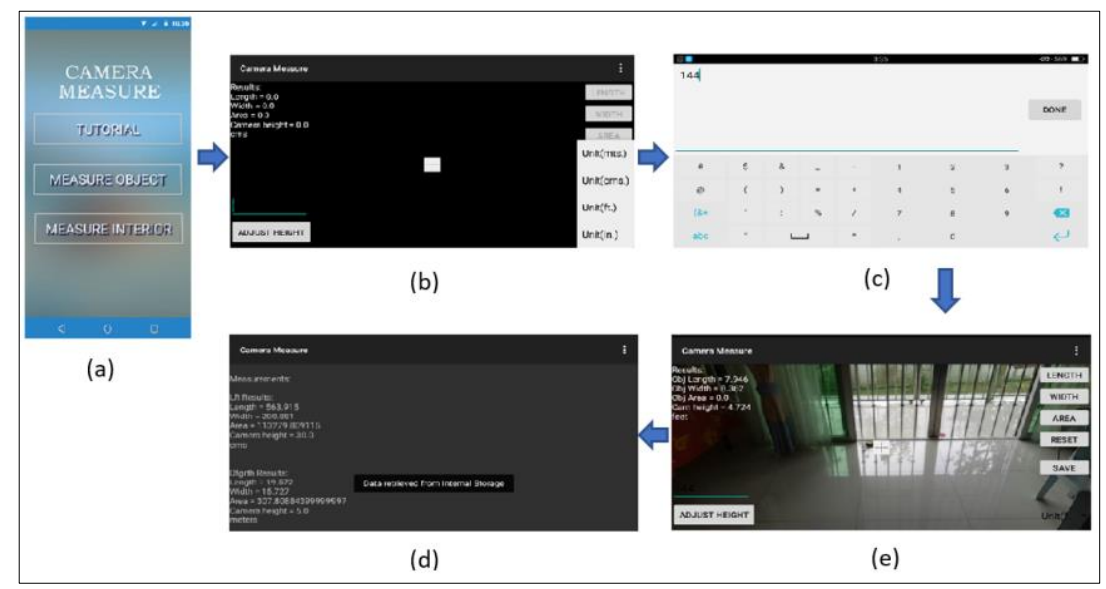

Fig. 5. Room Measurement Interfaces 
The interface is shown in Figure 5(b) will appear when the user clicks the Measure Interior button on the main interface, as seen in Figure 3(a). Users must first select the measurement unit to be used before entering the approximate height of the smartphone camera using the height button, as shown in Figures 5(b) and 5(c). Next, the user may select the length button, and move the camera from one point to another to measure the length. The reading will be captured automatically. The user can then select the width button and repeat the measurement process to determine the width of two points. The region will be measured automatically after the device has captured both length and width. Finally, the user may either choose to save the readings in the local internal storage by clicking the Save button or recapture the measurement by clicking the Reset button. Users can retrieve saved readings at any time using the menu button located at the top right of the screen.

\section{$5 \quad$ Evaluation Results}

In the post-survey, a questionnaire was generated by means of which the app review was carried out to decide if it meets the user's needs in terms of usability and functionality.

The usability questionnaire was sent to all users to test the developed app. Until the questionnaire was sent, we sent the users a final version of our app to their smartphones, to test our software, and to find any bugs. A few of them did not know how to install the app because the app is not available in the Google Play store. In addition, most of whom did not hold a university degree and served as private employees. As a result, users were given a brief description of the app installation. First, users were given 30 days to test the app and then fill out the survey form.

The questionnaire was designed on the basis of the System Usability Scale (SUS) [5], and its standard questions and calculations were used to obtain the results from the respondents. The SUS was determined by taking the average score from the survey result. The procedure used was to deduct one from the score of each oddnumbered question and deduct five from even-numbered questions. Such values were summed up and multiplied by 2.5 .

From the feedback received, the result approximately indicates that the developed app is acceptable to users. Nevertheless, not all users were completely committed to filling out the survey form; only 43 out of 50 respondents had completed the questionnaire. On average, the outcome of the SUS score is 78.08 percent based on the SUS calculation formula. Figure 8 shows the post-survey horizontal bar chart for user acceptance testing. 


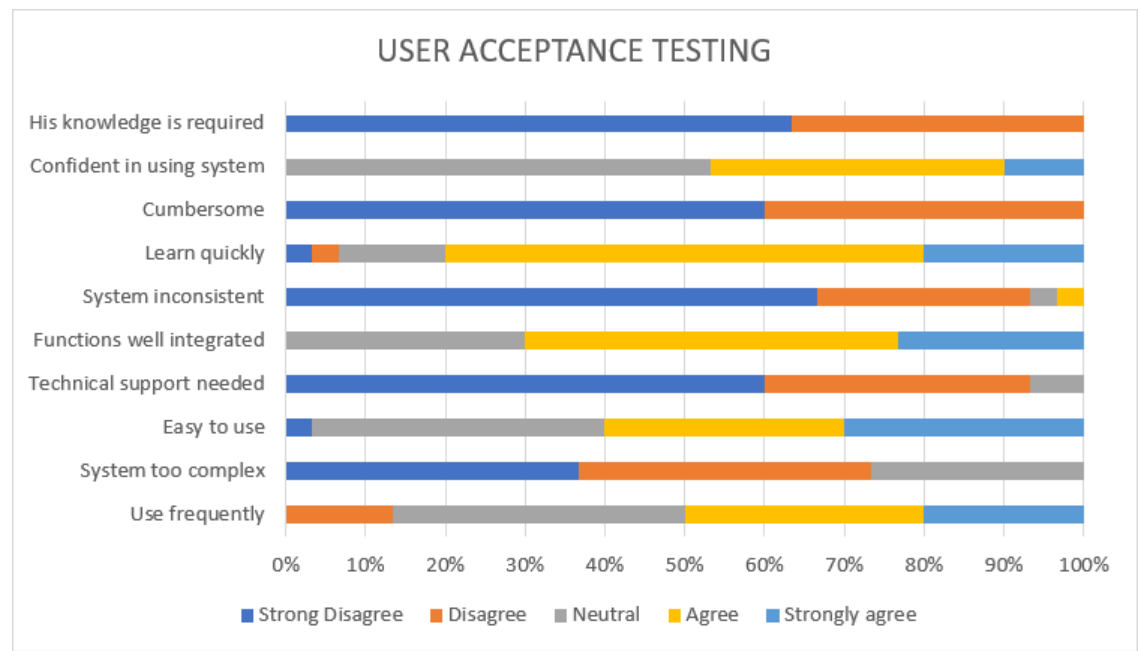

Fig. 6. System Usability Scale Test for the Measurement App

Figure 6 indicates that users who want to use the measurement app more often, are likely to find that the measurement app is not complicated, and is easy to use. A basic tutorial has been included in the app to guide users. In addition, the users decided that they did not need any technical support for the use of the measurement app and that no additional help or assistance was required during the measurement process. Usually, an assistant is needed when measuring tape is used. Users noticed that the essential measurement functions needed were well incorporated into the app, except for the angle measurement feature requested by a few interior designers.

The users agreed that the design of the measuring app was consistent, and they learned to use the measuring app quickly and easily. The users then decided that the app was not challenging to use, and they were comfortable using the app. Eventually, the users agreed that they did not know a lot until they got on with the app.

In addition to the usability test, both the post-survey functionality test and the findings are shown in Figure 7. 


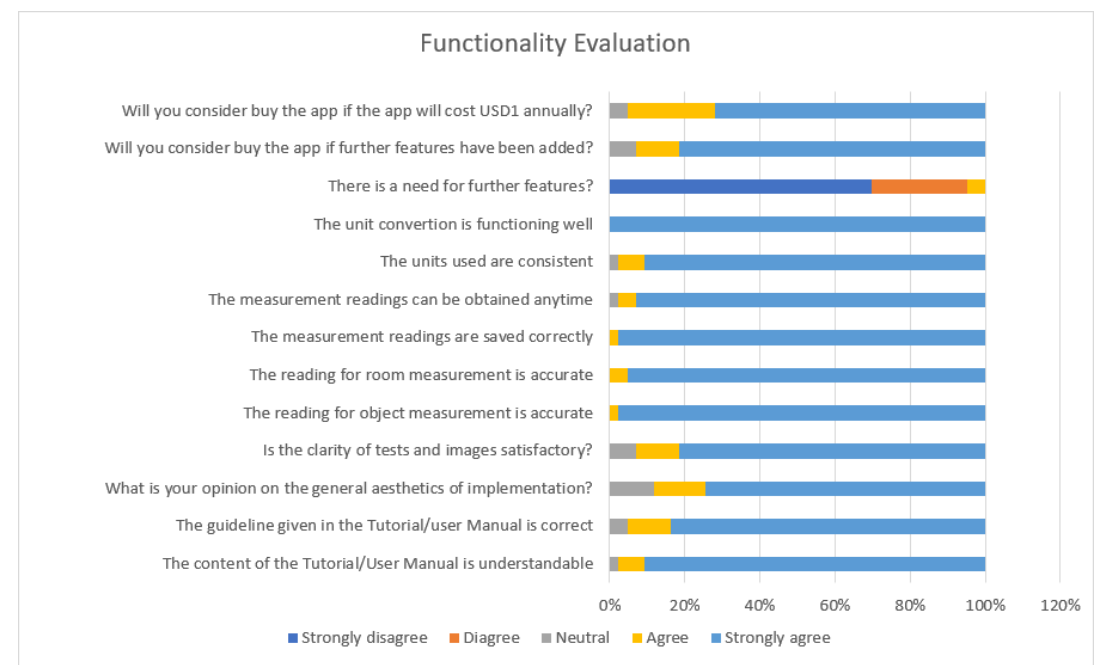

Fig. 7. System Functionality Scale Test for the Measurement App

Figure 7 shows users prefer a free app through the Google Play Store. Nevertheless, in our case, when asked about the purchase of the measuring app that we created, they replied positively if more features were added.

Then, in terms of design, through the replies have not received any negative feedback on the above questions that show us the positive feeling that users have experienced during the use of the application. And one of the most important goals during the development was achieved, where it was to be friendly and easy to use.

Also, in terms of design, no negative feedback has been provided through the responses, which show us the definite feeling that users have felt during the use of the app. So, the development goal was achieved, where it was to be friendly and easy to use.

In addition, we obtained essential results from the respondents on the functionality of the app. The app worked well to convert the various units used, save the readings correctly in the right directory, the readings for room and object measurements are accurate, and the tutorial info is correct. Few users have helped us understand some of their preferences and have inspired us to develop our application by adding a few new features if the app is no longer free of charge.

\section{$6 \quad$ Findings and Discussions}

Based on the results of the pre and post-surveys, a simple and easy to understand user manual is essential, especially for site contractors and piping workers. Most of them did not complete their study at secondary schools. Some of them are having difficulty reading and understand the English language. Perhaps the wordings should be replaced with a picture-based tutorial/ user manual. 
Measuring the dimension of an empty room could be simple using any tape or the proposed apps. But it is challenging if the room has existing furniture or object. Users must remove the furniture or object before the measurement is processed. Otherwise, the reading captured is not accurate.

A reference point is always an issue in using any measurement apps. The reference point must be first known and then inserted into the proposed app to acquire high accuracy measurement results. An alternative solution is required in the future to reduce this limitation.

Most of the measurement apps were designed based on its functionality, and hence some of the apps provided more than three features in a single app whilst some programmers integrated Augmented Reality technology into the apps. The apps are advanced, but it has been limited for those affordable for a high-end smartphone. However, the pre and post-results showed all workers are not affordable for high-cost devices. Furthermore, it is risky to use high-end smartphones in any construction site.

\section{Conclusion and Future Works}

Technology is evolving rapidly, and this enables more and more embedded systems to be placed in place to meet the increasing needs of everyday life, particularly in this era of Industrial Revolution 4.0. Smartphone technologies have evolved rapidly in recent years and have become essential items in daily life. Smartphones deliver so many apps to satisfy our needs, make our everyday lives easier, and make us productive and challenging to find in the past. The introduction of extra sensors enhanced mobile features such as artificial intelligence gaming [9][10], analysis of social media news using sentiment analysis [6][42], gamification [3][29], robot controller app [11], mobile e-learning [22][23][33], mobile e-government [4], etc. Some great deals of work have been done to address the above situations.

The theoretical implications of this research are the contributions to the designing of a mobile-based measurement tool, especially when considering that there is little research in this area. This study also provides more insight into the user requirement in developing apps. This paper addressed the concept and implementation of an Android Smartphone Measurement App prototype. It is a virtual measurement app to obtain the estimated length and width of a room by obtaining a reference scale from the user. Most existing apps in Google Play Store require high-end smartphones as augmented reality is used. But most of the actual users are not affordable for high-end smartphones. It, therefore, motivates us to build simple, low-end, and supportable measurement app.

The findings show that functionality is rather important than interactive interface design. End-user needs tutorial/guideline module, and they also prefer simple and easy to use features. They preferred reference point feature to be removed when using the measurement apps. Otherwise, the measurement reading is not accurate when a big room is measured.

In the future, researchers may consider improving the measurement app by including a laser light component to replace the reference point issue mentioned above. 
Furthermore, the image processing technique is required to automatically remove any object that blocks the view in a smartphone when a measurement is taken.

We have uploaded and posted the codes in the Github server. Programmers may access, edit, and further enhance the program. Codes are available at http:/github.com/etsen/etsen, and the guideline is available in a file called "readme.md."

\section{References}

[1] 3D Technologies. (2020). Moasure - the smart tape measure. Retrieved from https:// play.google.com/store/apps/details?id=com.Moasure. Moasure

[2] Anshari, M., \& Alas, Y. (2015). Smartphones Habits, Necessities, and Big Data Challenges. Journal of High Technology Management, 177-185. https://doi.org/10.1016/j.hitech.20 $\underline{15.09 .005}$

[3] Agustini, M., Yufiarti, Y., \& Wuryani, W. (2020). Development of Learning Media based on Android Games for Children with Attention Deficit Hyperactivity Disorder. International Journal of Interactive Mobile Technologies (iJIM), 14(06), 205-213. https://doi.org/ $\underline{10.3991 / i j i m . v 14 i 06.13401}$

[4] Alqaralleh, B. A., Al-Omari, A. H., \& Alksasbeh, M. Z. (2020). An Integrated Conceptual Model for m-Government Acceptance in Developing Countries: The Case Study of Jordan. International Journal of Interactive Mobile Technologies (iJIM), 14(06), 115-136. https ://doi.org/10.3991/ijim.v14i06.11449

[5] Bangor, A., Kortum, P.T., \& Miller, J.T. (2008). An Empirical Evaluation of the System Usability, International Journal of Human Computer. Interact, 574-594. https://doi.org/ $\underline{10.1080 / 10447310802205776}$

[6] Basri, S., Alfred, R., \& Chin, K.O. (2012). Automatic Spell Checker for Malay Blog. 2012 IEEE International Conference on Control System, Computing and Engineering, (pp. 506510). https://doi.org/10.1109/iccsce.2012.6487198

[7] Bento, N. (2016). Calling for Change? Innovation, Diffusion, and the Energy Impacts of Global Mobile Telephony. Journal of Energy Research \& Social Science, 84-100. https:// doi.org/10.1016/j.erss.2016.06.016

[8] CCubent. (2020). Photo Measures. Retrieved from https://play.google.com/store/apps/deta ils?id=com.bigbluepixel.photomeasures

[9] Chang, K.T., Ong, J.H., Teo, J., \& Chin, K.O. (2011). The Evolution of Gamebots for 3D First Person Shooter (FPS). 2011 Sixth International Conference on Bio-Inspired Computing: Theories and Applications, (pp. 21-26). https://doi.org/10.1109/bic-ta.2011.71

[10] Chin, K.O., Syukri, M.H., \& Tan, T.G. (2017). Design and Development of Novel Android 3D 3rd Person Shooting Game. International Conference on Computational Science and Technology, Singapore (pp. 302-313). Springer. https://doi.org/10.1007/978-981-10-8276$\underline{4 \_29}$

[11] Chin, K.O., \& Teo, J. (2010). Evolution and Analysis of Self-synthesized Minimalist Neural Controllers for Collective Robotics using Pareto Multi-objective Optimization. IEEE Congress on Evolutionary Computation, (pp. 1-7). https://doi.org/10.1109/cec.2010.55865 $\underline{37}$

[12] Dirk Farin. (2020). ImageMeter. Retrieved from https://play.google.com/store/apps/detai $\underline{\text { ls?id=de.dirkfarin.imagemeterprol }}$

[13] Farmis. (2020). GPS Fields Area Measure. Retrieved from https://play.google.com/store/ apps/details?id=lt.noframe.fieldsareameasure.adfree 
[14] Gamma Play. (2020). Bubble Level. Retrieved from https://play.google.com/store/apps/det ails?id=com.gamma.bubblelevel

[15] Google Developer. (2020). Android Studio. Retrieved from https://developer.android.com/ $\underline{\text { studio }}$

[16] Google LLC. (2020). Measure. Retrieved from https://play.google.com/store/apps/details? id=com.google.tango.measure

[17] Grymala. (2020a). Ruler App - Camera. Retrieved from https://play.google.com/store/ap ps/details?id=com.grymala.photoruler

[18] Grymala. (2020b). AR Ruler App. Retrieved from https://play.google.com/store/apps/deta ils?id=com.grymala.aruler

[19] Grymala. (2020c). AR Plan 3D Ruler. Retrieved from https://play.google.com/store/apps/ details?id=com.grymala. arplan

[20] Holzmann, C., \& Hochgatterer, M. (2012). Measuring Distance with Mobile Phones using Single-Camera Stereo Vision. 2012 32nd International Conference on Distributed Computing Systems Workshops (ICDCSW), (pp. 88-93). https://doi.org/10.1109/icdcsw.2012.22

[21] Interactive Specialized Software Tools. (2020). Distance Laser Measure. Retrieved from https://play.google.com/store/apps/details?id=com.interactivespecializedsoftware.distanc

[22] Kalogiannakis, M., \& Papadakis, S. (2017). Combining mobile technologies in environmental education: a Greek case study. International Journal of Mobile Learning and Organisation, 11(2), 108-130.

[23] Kalogiannakis, M., Nirgianaki, G. M., \& Papadakis, S. (2018). Teaching magnetism to preschool children: The effectiveness of picture story reading. Early Childhood Education Journal, 46(5), 535-546.

[24] LKE Tech. (2020). Fields Area Measure. Retrieved from https://play.google.com/store/ apps/details?id=com.lketech.maps.area.calculator

[25] Losa-Iglesias, M. E., Losa, K. B. D. B., López-López, D., Jimenez-Fernandez, R., CorralLiria, I., Vazquez, R. R., ... \& Vallejo, R. B. D. B. (2019). The Usability of a Heartbeat Measuring Mobile Phone app: an Observational Study. Journal of medical systems, 43(7), 226. https://doi.org/10.1007/s10916-019-1367-5

[26] Markhordevelopers. (2020). GPS Land Area Calculator. Retrieved from https://play.goog le.com/store/apps/details?id=markhor.gps.area.measurement

[27] Mota, J. M., Ruiz-Rube, I., Dodero, J. M., \& Arnedillo-Sánchez, I. (2018). Augmented Reality Mobile App Development for All. Journal of Computers \& Electrical Engineering, 65, 250-260. https://doi.org/10.1016/j.compeleceng.2017.08.025

[28] Nakhon Phagdeechat. (2020). Angle Meter Pro Plus. Retrieved from https://play.google. com/store/apps/details?id=iyok.com.anglemeterproplus

[29] Ng, C.H., Niew, S.H., Chin, K.O., \& Teo, J. (2011). Infinite Mario Bross AI using Genetic Algorithm. 2011 IEEE Conference on Sustainable Utilization and Development in Engineering and Technology (STUDENT), (pp. 85-89). https://doi.org/10.1109/student.2011.60 $\underline{89330}$

[30] New Marathon Ltd. (2020). GPS Area Measure. Retrieved from https://play.google.com/s tore/apps/details?id=com.marathon.gps.fieldarea

[31] Nikola Kosev. (2020). Ruler. Retrieved from https://play.google.com/store/apps/details?id =net.kosev.rulering

[32] NixGame. (2020). Ruler. Retrieved from https://play.google.com/store/apps/details?id= org.nixgame.ruler

[33] Papadakis, St., \& Kalogiannakis, M. (2017). Mobile educational applications for children. What educators and parents need to know. 
International Journal of Mobile Learning and Organisation (Special Issue on Mobile Learning Applications and Strategies), 11(3), 256-277.

[34] Ponica Media. (2020). Ruler, Bubble Level, Vernier Caliper, Measurement. Retrieved from https://play.google.com/store/apps/details?id=com.ponicamedia.android.ruler

[35] Shyam Barange. (2020). AR Ruler - Tape Measure. Retrieved from https://play.google.com/store/apps/details?id=com.ShyamBarange.ARCoreMeasure

[36] Sjöberg, J., \& Persson, M. (2014). Optical Pedometer: A New Method for Distance Measuring using Camera Phones. Retrieved from https://www.semanticscholar.org/paper/Opt ical-Pedometer-\%3A-A-new-method-for-distance-using-Sj\%C3\%B6berg-Persson/b852fe2 4ea2b81fecffa1e22b21bd6a29b361c0b

[37] Smart Tools Co. (2020a). Ruler. Retrieved from https://play.google.com/store/apps/details ? id=kr.sira.ruler

[38] Smart Tool Co. (2020b). Smart Measure Pro. Retrieved from https://play.google.com/sto re/apps/details?id=kr.aboy.measure

[39] Smart Tool Co. (2020c). Smart Protractor. Retrieved from https://play.google.com/store/ap ps/details?id=kr.sira.protractor

[40] Smart Tool Factory. (2020). Angle Meter. Retrieved from https://play.google.com/store/ap ps/details?id=com.stfactory.anglemeter

[41] Susilo, Y. O., \& Liotopoulos, F. K. (2018). Measuring Door-to-Door Journey Travel Satisfaction with a Mobile Phone App. In Quality of Life and Daily Travel (pp. 119-138). Springer, Cham. https://doi.org/10.1007/978-3-319-76623-2 7

[42] Tan, L.I., Phang, W.S., Chin, K.O., \& Anthony, P. (2015). Rule-based Sentiment Analysis for Financial News. 2015 IEEE International Conference on Systems, Man, and Cybernetics, (pp. 1601-1606). https://doi.org/10.1109/smc.2015.283

[43] Tasmanic Editions. (2020). CamToPlan. Retrieved from https://play.google.com/store/ apps/details?id=com.tasmanic.camtoplanfree

[44] Van Thanh, P., Thi, A. D. N., Thuy, Q. T. T., Phuong, D. C. T., Mau, V. H., \& Tran, D. T. (2017, October). A Novel Step Counter Supporting for Indoor Positioning Based on Inertial Measurement Unit. In 2017 7th International Conference on Integrated Circuits, Design, and Verification (ICDV) (pp. 69-74). IEEE. https://doi.org/10.1109/icdv.2017.81886 41

[45] Xalpha Lab. (2020). Ruler. Retrieved from https://play.google.com/store/apps/details?id=f r.ecp.ruler.app

\section{Authors}

Elcy Tsen is a research assistant at Faculty of Computing and Informatics, Universiti Malaysia Sabah, from 2017-2020.

Chin Kim On is a Malaysia computer scientist, Senior Member of IEEE, and Computational Thinking educator at Faculty of Computing and Informatics, Universiti Malaysia Sabah, Kota Kinabalu, Sabah. His research interests include artificial neural networks, image processing and its applications, and sentiment analysis.

Rayner Alfred holds a $\mathrm{PhD}$ degree in Computer Science from York University (United Kingdom), a Master degree in Computer Science from Western Michigan University, Kalamazoo (USA) and a Computer Science degree from Polytechnic University of Brooklyn, New York (USA) where he was the recipient of the Myron M. 
Rosenthal Academic Achievement Award for the outstanding academic achievement in Computer Science in 1994.

Mohd Hanafi Ahmad Hijazi is an Associate Professor at the Faculty of Computing and Informatics, Universiti Malaysia Sabah, has research interest in data mining, knowledge discovery in healthcare and computer vision.

Tan Tse Guan received the Bachelor of Computer Science (Hons) in Software Engineering, the Master of Science in Artificial Intelligence and the Doctor of Philosophy in Computer Science from Universiti Malaysia Sabah (UMS), Malaysia, in 2006, 2008 and 2013, respectively. Currently, he is the Deputy Dean of Research, Innovation and Postgraduate at the Faculty of Creative Technology and Heritage, Universiti Malaysia Kelantan (UMK), Malaysia.

Article submitted 2020-05-04. Resubmitted 2020-08-17. Final acceptance 2020-08-17. Final version published as submitted by the authors. 